\title{
Conhecimentos de médicos anestesiologistas sobre cuidados em anestesiologia nos pacientes com COVID-19
}

\author{
Anesthesiologists knowledge about anesthesiology care in patients with COVID-19 \\ Conocimiento de anestesiólogos en el manejo anestésico de los pacientes con COVID-19
}

Recebido: 25/08/2021 | Revisado: 30/08/2021 | Aceito: 06/09/2021 | Publicado: 07/09/2021

Emily Maria Gambetta

ORCID: https://orcid.org/0000-0002-3869-7419 Centro Universitário da Fundação Assis Gurgacz, Brasil

E-mail: emily.gambetta@gmail.com

Maurício Henrique Zanini Centenaro

ORCID: https://orcid.org/0000-0002-2981-1304 Centro Universitário da Fundação Assis Gurgacz, Brasil E-mail: maucentenaro@gmail.com

Mauro da Cruz Assad Monteiro

ORCID: https://orcid.org/0000-0002-3487-7092 Centro Universitário da Fundação Assis Gurgacz, Brasil E-mail:mauro assad@hotmail.com

Larissa Kerr de Araújo Sodré

ORCID: https://orcid.org/0000-0003-3877-5218 Centro Universitário da Fundação Assis Gurgacz, Brasil E-mail: larissa kerr@hotmail.com

Ivan Roberto Bonotto Orso

ORCID: https://orcid.org/0000-0003-4034-8821 Centro Universitário da Fundação Assis Gurgacz, Brasil E-mail: ivan@gastro.com.br

\begin{abstract}
Resumo
A COVID-19 é causada por um betacoronavírus denominado SARS-CoV-2. O quadro clínico consiste em sinais e sintomas inespecíficos como febre, tosse seca, dispneia, mialgia, fadiga, cefaleia, anosmia e sintomas gastrointestinais. A recomendação de manejo invasivo de vias aéreas em pacientes com COVID-19 é por intubação orotraqueal de sequência rápida. Como esse e outros procedimentos anestesiológicos são geradores de aerossóis, é fundamental a proteção dos profissionais de saúde com Equipamentos de Proteção Individual (EPIs). Dessa forma, este estudo buscou avaliar a utilização de EPIs e também o conhecimento de médicos anestesiologistas no manejo de pacientes com COVID-19. Dos 53 profissionais entrevistados, $13(24,5 \%)$ relataram que em pelo menos um procedimento não tiveram acesso aos EPIs necessários para sua segurança. Esse é possivelmente um dos fatores relacionados a elevada prevalência de adoecimento por COVID-19 na amostra de profissionais participantes do estudo (30,2\%). Além disso, 28,3\% dos participantes da pesquisa calcularam incorretamente o volume corrente de uma paciente fictícia. Assim, conclui-se que as condutas realizadas por esses profissionais são parcialmente adequadas ao manejo perioperatório de pacientes com COVID-19 de acordo com as literaturas consultadas. Portanto, são essenciais medidas de educação continuada para ampliar a assertividade dos médicos nas condutas realizadas. Ademais, as instituições de saúde devem prover EPIs necessários aos profissionais de saúde para reduzir a chance de infecção destes trabalhadores.
\end{abstract}

Palavras-chave: COVID-19; Anestesiologia; Equipamento de proteção individual; Saúde do trabalhador.

\begin{abstract}
COVID-19 is caused by SARS-CoV-2 betacoronavirus. The clinical manifestations consist of nonspecific signs and symptoms such as fever, dry cough, dyspnea, myalgia, fatigue, headache, anosmia and gastrointestinal symptoms. The recommendation for invasive airway management in patients with COVID-19 is using rapid sequence orotracheal intubation. This and other anesthetic procedures generates aerosols, so its essential to protect healthcare professionals with Personal Protective Equipment (PPE). Thus, this study sought to evaluate the use of PPE and also the knowledge of anesthesiologists in the management of patients with COVID-19. Of the 53 professionals interviewed, 13 (24,5\%) reported that they did not had access to the PPE necessary for their safety in at least one procedure. This is a possibly factor related to the high prevalence of COVID-19 illness in the sample of professionals participating in the study $(30,2 \%)$. In addition, $28,3 \%$ of the survey participants incorrectly calculated the tidal volume of a fictious patient. Thus, we concluded that procedures performed by these professionals are partially adequate for COVID-19 patients in perioperative management according to the literature consulted. Therefore, continued education measures are essential
\end{abstract}


to increase doctor's assertiveness in the conducts. Furthermore, health institutions must provide necessary PPE to healthcare professionals to reduce the chance of infection of these workers.

Keywords: COVID-19; Anesthesiology; Individual protection equipment; Worker's health.

\section{Resumen}

COVID-19 es causado por un betacoronavírus llamado SARS-CoV-2. El cuadro clínico consiste en signos y síntomas inespecíficos como fiebre, tos seca, disnea, mialgia, fatiga, cefalea, anosmia y síntomas gastrointestinales. La recomendación para el manejo invasivo de las vías respiratorias en pacientes con COVID-19 es mediante intubación orotraqueal de secuencia rápida. Como éste y otros procedimientos anestésicos son generadores de aerosoles, es fundamental proteger los profesionales de salud con Equipos de Protección Personal (EPPs). Así, este estudio buscó evaluar el uso de EPPs y también conocimiento de los anestesiólogos en el manejo de pacientes con COVID-19. De los 53 profesionales entrevistados, 13 (24,5\%) informaron que en al menos un procedimiento no tuvieron acceso al EPP necesario para su seguridad. Este es posiblemente uno de los factores relacionados con la alta prevalencia de enfermedad por COVID-19 en la muestra de profesionales participantes del estudio (30,2\%). Además, 28,3\% de los participantes calculó incorrectamente el volumen corriente de un paciente ficticio. Así, se concluye que los procedimientos realizados por estos profesionales son parcialmente adecuados para el manejo perioperatorio de los pacientes con COVID-19 según la literatura consultada. Por tanto, las medidas de educación continua son fundamentales para incrementar el asertividad de los médicos en las conductas realizadas. Además, las instituciones de salud deben proporcionar el EPP necesario a los profesionales de salud para reducir la posibilidad de infección de estos trabajadores.

Palabras clave: COVID-19; Anestesiología; Equipo de protección individual; Salud del trabajador.

\section{Introdução}

Os primeiros casos de Coronavirus Disease-2019 (COVID-19) sugiram em-Wuhan (China) (Zhu et al, 2020). A Covid19 tem como agente etiológico um betacoronavirus denominado SARS-CoV-2, cujo diagnóstico pode ser realizado por meio de testes moleculares e imunoensaios (Vandenberg et al, 2021). A sensibilidade dos exames moleculares é dependente da qualidade da amostra (Loeffelhoz et al, 2020; Lieberman et al, 2020), sendo de 93\% no fluido broncoalveolar, 72\% na expectoração e de $63 \%$ nos swabs nasofaríngeos (Wang et al, 2020).

Os testes moleculares são recomendados nos primeiros sete dias dos sintomas (Magno et al, 2020). Após esse período são recomendados testes rápidos (imunocromatografia) ou ELISA para detecção de anticorpos IgM e/ou IgG (Magno et al, 2020). O IgM leva cerca de cinco dias para ser detectado após o início dos sintomas, enquanto o IgG torna-se detectável mais tardiamente (Guo et al, 2020). A principal desvantagem dos testes de detecção de anticorpos são a possibilidade de reação cruzada com outros vírus, principalmente os da mesma família viral (Petherick, 2020).

O quadro clínico consiste em sinais e sintomas inespecíficos como febre, tosse seca, dispneia, mialgia, fadiga, cefaleia, dispneia, anosmia e sintomas gastrointestinais como diarreia (Wiersinga et al, 2020). As complicações mais comuns são pneumonia, síndrome respiratória aguda grave (SARS), injúria hepática aguda, injúria cardíaca, eventos tromboembólicos, injúria renal e manifestações neurológicas como rebaixamento do nível de consciência (Wiersinga et al, 2020).

Dessa forma, como o manejo anestésico para intubação orotraqueal apresenta diversas especificidades, especialmente em pacientes com COVID-19, esse estudo analisou o conhecimento de médicos anestesiologistas nas condutas em pacientes com e sem essa patologia.

\section{Metodologia}

Trata-se de um estudo observacional, prospectivo e de caráter quantitativo, realizado através do preenchimento de questionários por médicos anestesiologistas com atuação no estado do Paraná. A pesquisa foi aprovada pelo Comitê de Ética em Pesquisa com Seres Humanos do Centro Universitário da Fundação Assis Gurgacz com número CAAE 39852320.1.000.5219. Como base metodológica utilizou-se o trabalho de Pereira et al (2018).

Considerando a situação de pandemia por Sars-CoV-2, foi disponibilizada para os participantes da pesquisa, além da versão impressa, a possibilidade de preenchimento virtual dos questionários por meio do Google Forms. 
Os médicos anestesiologistas foram questionados quanto: 1) idade, há quanto tempo trabalha com anestesiologia, em quantas instituições trabalha e qual o vínculo empregatício; 2) se já testou positivo para COVID-19; 3) se realizou ou participou de procedimentos geradores de aerossóis e quais foram; 4) quais equipamentos de proteção individual podem ser reutilizados; 5) se teve acesso aos equipamentos de proteção individual em todos os procedimentos nos quais participou e motivos de não utilização; 6) conhecimento no manejo das vias aéreas de pacientes confirmados ou suspeitos de COVID-19 e a conduta realizada se impossibilidade de intubação orotraqueal; 7) conhecimento sobre bloqueador neuromuscular relacionado a sequência rápida de intubação orotraqueal; 8) conhecimento sobre ventilação mecânica.

A análise estatística para determinação das frequências absolutas e relativas das categorias analisadas foi realizada no software RStudio na versão 1.4.1103.

\section{Resultados e Discussão}

De acordo com Tang e Wang (2020), a recomendação de manejo invasivo de vias aéreas em pacientes com COVID-19, como intubação orotraqueal de sequência rápida, se deve ao fato de que formas não invasivas de ventilação, como oxigenação com bolsa-válvula-máscara aumentam a geração de aerossóis das secreções aéreas (Meng et al, 2020; Anesi, 2021). Como a intubação é o procedimento com maior risco de dispersão de aerossóis em pacientes com COVID-19 (Cheung et al, 2020; Feldman et al, 2020; El-Boghdadly et al, 2020) é fundamental a proteção dos profissionais de saúde com Equipamentos de Proteção Individual (EPIs) que inclui avental cirúrgico, lentes de proteção com selo completo, máscaras N95, pijama, luvas e botas cirúrgicas (Velázquez et al, 2020).

Além da utilização dos EPIs, é recomendado que o procedimento de intubação seja realizado em salas de isolamento de infecções respiratórias transportadas pelo ar (SIIA) com pressão negativa, se possível (Anesi, 2021; Tang e Wang, 2020; Kim et al, 2020). Em estudo realizado por Lee et al (2020) a instalação dessas salas foi essencial durante a pandemia.

Outras recomendações no manejo das vias aéreas em pacientes com COVID-19 incluem a preferência pela intubação orotraqueal em detrimento da via supra-glótica por efetivamente "selar" a via aérea e prevenir disseminação viral, o uso de equipamentos descartáveis sempre que possível, intubação realizada pelo profissional mais experiente e uso do laringoscópio que o médico achar mais confortável para realizar intubação mais rapidamente (London, 2021). O uso do videolaringoscópio geralmente é preferido pois amplia as chances de sucesso em pacientes com via aérea difícil (Schumacher et al, 2020). Em caso de necessidade de ventilação de resgate, recomenda-se utilização da via supraglótica em detrimento de ventilação por máscara por possibilitar intubação pelo dispositivo supra-glótico (London, 2021).

A Tabela 1 resume informações referentes aos médicos participantes da pesquisa. A maioria apresenta idade entre $21 \mathrm{e}$ 40 anos (90,5\%), atua na área de anestesiologia há até 5 anos $(62,2 \%)$, trabalha em mais que duas instituições (81,1\%), em grupos de anestesiologistas (56,6\%) e não teve diagnóstico molecular ou via teste rápido de COVID-19 (69,8\%). 
Tabela 1: Informações relatadas pelos médicos anestesiologistas participantes da pesquisa referentes a idade, tempo de trabalho na área de anestesiologia, em quantas instituições trabalha, tipo de vínculo empregatício e diagnóstico confirmado de COVID-19 (n=53).

\begin{tabular}{lcc}
\hline Idade & $\begin{array}{c}\text { Frequência } \\
\text { Absoluta }\end{array}$ & Frequência Relativa \\
\hline $21-30$ anos & 14 & $26,4 \%$ \\
$31-40$ anos & 34 & $64,1 \%$ \\
$41-50$ anos & 1 & $1,9 \%$ \\
$51-60$ anos & 2 & $3,8 \%$ \\
$61-70$ anos & 2 & $3,8 \%$ \\
Há quanto tempo trabalha com anestesiologia & & \\
$<1$ ano & 5 & $9,4 \%$ \\
$1-5$ anos & 28 & $52,8 \%$ \\
$6-10$ anos & 14 & $26,4 \%$ \\
$11-20$ anos & 2 & $3,8 \%$ \\
$21-30$ anos & 1 & $1,9 \%$ \\
$31-40$ anos & 3 & $5,7 \%$ \\
Em quantas instituições trabalha & & \\
$0-1$ instituições & 10 & $18,9 \%$ \\
$2-3$ instituições & 21 & $39,6 \%$ \\
$\geq 4$ instituições & 22 & $41,5 \%$ \\
Vínculo empregatício & & \\
Profissional liberal & 16 & $30,2 \%$ \\
Vinculado a grupo de anestesiologistas & 30 & $56,6 \%$ \\
Emprego público & 5 & $9,4 \%$ \\
Residente & 2 & $3,8 \%$ \\
Teve diagnóstico confirmado de COVID-19? & & $30,2 \%$ \\
Sim & 16 & $69,8 \%$ \\
Não & 37 &
\end{tabular}

Fonte: Autores (2021).

Entre os profissionais participantes do estudo, $83 \%$ relataram participar ou realizar procedimentos geradores de aerossóis em pacientes com COVID-19, que provavelmente são a principal fonte de disseminação viral especialmente em unidades de saúde (El-Wahab et al, 2020) (Tabela 2). Os procedimentos geradores de aerossóis que os anestesiologistas mais relataram participar ou realizar em enfermos com COVID-19 foram intubação orotraqueal (79,2\%), posicionamento de ventilação sob máscara $(54,7 \%)$ e cirurgias envolvendo perfuração $(41,5 \%)$ (Tabela 2).

A utilização da técnica de Intubação em Sequência Rápida em pacientes com COVID-19, que apresenta benefícios na comparação com outras técnicas pela menor produção de aerossóis contaminantes (London 2021; Anesi, 2021) é utilizada por $62,3 \%$ dos anestesiologistas no manejo da via aérea avançada (Tabela 2). Na falha desta técnica, 77,3\% dos médicos interrogados utilizaram dispositivo supra-glótico e $22,7 \%$ via aérea cirúrgica. 
Tabela 2: Informações relatadas de participação ou realização de procedimentos geradores de aerossóis em pacientes com COVID-19 e descrição destes procedimentos por médicos anestesiologistas participantes da pesquisa.

\begin{tabular}{lcc}
\hline $\begin{array}{l}\text { Realizou ou participou de procedimentos } \\
\text { geradores de aerossóis em pacientes com }\end{array}$ & $\begin{array}{c}\text { Frequência } \\
\text { Absoluta }\end{array}$ & Frequência Relativa \\
COVID-19? & 44 & $83 \%$ \\
\hline Sim & 9 & $17 \%$ \\
Não & 7 & \\
Caso a resposta for afirmativa, quais procedimentos realizou ou participou? & \\
Broncoscopia rígida & 17 & $13,2 \%$ \\
Traqueostomia & 22 & $32,1 \%$ \\
Cirurgias envolvendo perfuração & 42 & $41,5 \%$ \\
Intubação orotraqueal & 7 & $79,2 \%$ \\
Intubação por fibra óptica & 29 & $13,2 \%$ \\
Posicionamento de ventilação sob & & $54,7 \%$ \\
máscara & 9 & $17 \%$ \\
Posicionamento de cânula nasal de alto & & \\
fluxo & 15 & $28,3 \%$ \\
Aspiração de secreções & 15 & $28,3 \%$ \\
Reanimação cardiopulmonar & & \\
Qual técnica de intubação tem utilizado em pacientes sem diagnóstico confirmado de COVID- \\
19 ou suspeitos? \\
Intubação em Sequência Rápida & 33 & $62,3 \%$ \\
Outras técnicas & 20 & $37,7 \%$ \\
Na impossibilidade de intubação orotraqueal qual conduta tomou (n=44) & \\
Dispositivo supra-glótico & 34 & $77,3 \%$ \\
Via aérea cirúrgica & 10 & $22,7 \%$ \\
\hline
\end{tabular}

Fonte: Autores (2021).

Para a segurança dos profissionais de saúde é essencial a disponibilização e utilização correta dos equipamentos de proteção individual (EPIs). Estudos relatam que profissionais que utilizam corretamente EPIs apresentam menor risco de desenvolvimento de doenças respiratórias e influenza-like (Offeddu, 2017). Contudo, 24,5\% dos médicos participantes da pesquisa relataram que não tiveram acesso, em pelo menos um procedimento que realizou, a EPIs necessários para sua segurança (Tabela 3). Destes profissionais, 92,3\% relataram que não tiveram acesso por escassez de EPIs. Outros trabalhos mostraram que a falta destes equipamentos em unidades de saúde se agravou com a pandemia (Breazzano et al, 2020).

Outro fator relevante para a biossegurança dos profissionais envolvidos em ambientes cirúrgicos é a presença da sala de isolamento de infecções respiratórias transportadas pelo ar (SIIA). Embora exista recomendação de realizar todos os procedimentos geradores de aerossóis nessas salas (Ruetzler et al, 2020), para reduzir possibilidade de contaminação dos profissionais, apenas $22,6 \%$ dos médicos declararam trabalhar em hospitais que contam com SIIA (Tabela 3). 
Tabela 3: Informações referentes a acesso de Equipamentos de Proteção Individual (EPIs), motivos para não ter acesso aos EPIs e existência de sala de isolamento de infecções respiratórias transportadas pelo (SIIA) nas instituições que os médicos anestesiologistas trabalham.

\begin{tabular}{lcc}
\hline $\begin{array}{l}\text { Teve acesso a Equipamentos de Proteção } \\
\text { Individual (EPIs) necessários para sua segurança } \\
\text { em todos os procedimentos que participou? }\end{array}$ & $\begin{array}{c}\text { Frequência } \\
\text { Absoluta }\end{array}$ & Frequência Relativa \\
\hline Sim & 40 & $75,5 \%$ \\
Não & 13 & $24,5 \%$ \\
Por qual motivo não teve acesso? (n=13) & 12 & $92,3 \%$ \\
$\quad$ Escassez de equipamentos & 1 & $7,7 \%$ \\
$\quad$ Recusa da instituição em disponibilizar & & \\
Na instituição em que você trabalha existe sala cirúrgica de isolamento de infecções respiratórias \\
transportadas pelo ar (SIIA)? \\
$\quad$ Sim \\
Não
\end{tabular}

Fonte: Autores (2021).

$\mathrm{Na}$ Tabela 4 foram avaliados conhecimentos gerais e relacionados a pacientes com COVID-19 dos médicos anestesiologistas. O Cisatracúrio, por suas características farmacodinâmicas e farmacocinéticas, demora mais tempo para ter ação na comparação com drogas como o Rocurônio (Lee, 2013) e, dessa forma, não é adequada sua utilização na intubação orotraqueal em sequência rápida. A pergunta que abordava este conceito foi respondida corretamente por 96,2\% dos anestesiologistas participantes do estudo (Tabela 4).

Já o cálculo do volume corrente é essencial para a adequada oxigenação do paciente, para evitar lesões pulmonares e liberação de mediadores inflamatórios pulmonares (The Acute Respiratory Distress Syndrome Network, 2000). Ao perguntar se $300 \mathrm{~mL}$ de volume corrente seria adequado em uma paciente feminina com $90 \mathrm{~kg}$ e $155 \mathrm{~cm}$ de altura, $28,3 \%$ dos profissionais responderam equivocadamente que não. Nesse caso, por se tratar de uma paciente com obesidade, seria necessário calcular o peso ideal inicialmente, para em seguida calcular o volume corrente.

Equipamentos como Face Shield, máscara N95 e óculos de proteção, podem ser reutilizados, embora esta prática esteja associada a maiores níveis de contaminação no caso dos óculos (Lange, 2014; Sesesirikachorn et al, 2020; Armijo et al, 2020). Mais que $90 \%$ dos médicos participantes da pesquisa responderam corretamente que esses materiais eram reutilizáveis e 15,1\% e $37,7 \%$ citaram, equivocadamente, que máscaras cirúrgicas e aventais cirúrgicos podem, respectivamente, ser reutilizados. 
Tabela 4: Informações relacionadas as perguntas de avaliação do conhecimento de médicos anestesiologistas.

\begin{tabular}{lcc}
\hline $\begin{array}{l}\text { Cisatracúrio é um bloqueador neuromuscular } \\
\text { adequado a indução em sequência rápida para } \\
\text { intubação orotraqueal? }\end{array}$ & $\begin{array}{c}\text { Frequência } \\
\text { Absoluta }\end{array}$ & $\begin{array}{c}\text { Frequência Relativa } \\
\text { Sim }\end{array}$ \\
\hline Não & 2 & $3,8 \%$ \\
No caso de um paciente feminina com $90 \mathrm{~kg}$ e $155 \mathrm{~cm}$ de altura, seria adequado ofertar 300mL de \\
ar a cada ciclo respiratório? \\
Sim & 38 & $71,7 \%$ \\
Não & 15 & $28,3 \%$ \\
Quais equipamentos de proteção individual podem ser reutilizados? & \\
Máscara cirúrgica & 8 & $15,1 \%$ \\
Máscara N95 & 48 & $90,6 \%$ \\
Face Shields & 51 & $96,2 \%$ \\
Avental & 20 & $37,7 \%$ \\
Óculos de proteção & 51 & $96,2 \%$ \\
\hline
\end{tabular}

Fonte: Autores (2021).

Esse estudo buscou, além de verificar a utilização de equipamentos de proteção individual e presença de salas de isolamento de infecções respiratórias transportadas pelo ar (SIIA) nas instituições em que os profissionais trabalham, avaliar o conhecimento de médicos anestesiologistas nas condutas em pacientes com e sem essa patologia. Observou-se que a maioria respondeu corretamente às perguntas, contudo 28,3\% (Tabela 4) erraram o cálculo do volume corrente. Dessa forma, vê-se que são necessárias medidas de educação continuada para ampliar a assertividade dos profissionais nas condutas realizadas.

Ainda, a elevada prevalência de COVID-19 entre médicos anestesiologistas (30,2\%), conforme destacado pela Tabela 1 pode ser decorrente da elevada exposição ocupacional pois $83 \%$ deles relataram realização ou participou em procedimentos geradores de aerossóis em pacientes com COVID-19 (Tabela 2).

Outro fator que pode ter contribuído para as contaminações pelo Sars-CoV-2 foi a falta de acesso a Equipamentos de Proteção Individual (EPIs) necessários para segurança em pelo menos um procedimento, fato que foi relatado por $24,5 \%$ dos profissionais entrevistados.

Além disso, destaca-se a baixa penetração de SIIAs nas instituições em que os médicos trabalham. Essas salas, por conterem pressão negativa, propiciam maior segurança aos profissionais de saúde que necessitam realizar procedimentos geradores de aerossóis em pacientes com COVID-19.

\section{Conclusão}

O presente trabalho cumpriu o objetivo de descrever e analisar o conhecimento de médicos anestesiologistas sobre manejo perioperatório de pacientes com suspeita de COVID-19 e também de verificar medidas de prevenção e uso de EPIs por parte das equipes de anestesiologia durante a pandemia por COVID-19. As condutas realizadas por médicos anestesiologistas atuantes no estado do Paraná foram comparadas com as condutas recomendadas na literatura internacional e foi possível concluir que estavam parcialmente adequadas a literatura consultada.

Futuros estudos podem abordar esta temática em estudos multicêntricos, verificando em quais hospitais as condutas e o conhecimento dos médicos anestesiologistas referente ao manejo perioperatório de pacientes com suspeita de COVID-19 estão mais adequados com relação a literatura nacional e internacional. 


\section{Referências}

Abd El-Wahab, E. W., Eassa, S. M., Metwally, M., Al-Hraishawi, H., \& Omar, S. R. (2020). SARS-CoV-2 transmission channels: A review of the literature. MEDICC Review, 22(4), 61-69. https://doi.org/10.37757/MR2020.V22.N4.3

Anesi, G. L. (2021). COVID-19: Critical care and airway management issues. UpToDate. https://www.uptodate.com/contents/covid-19-critical-care-andairway-management-issues

ARDS Network. (2000). Ventilation With Lower Tidal Volumes As Compared With Traditional Tidal Volumes for ALI and the ARDS. The New England Journal of Medicine, 342(18), 1301-1308.

Armijo, P. R., Markin, N. W., Nguyen, S., Ho, D. H., Horseman, T. S., Lisco, S. J., \& Schiller, A. M. (2021). 3D printing of face shields to meet the immediate need for PPE in an anesthesiology department during the COVID-19 pandemic. American Journal of Infection Control, 49(3), 302-308. https://doi.org/10.1016/j.ajic.2020.07.037

Breazzano, M. P., Shen, J., Abdelhakim, A. H., Glass, L. R. D., Horowitz, J. D., Xie, S. X., De Moraes, C. G., Chen-Plotkin, A., \& Chen, R. W. S. (2020). New York City COVID-19 resident physician exposure during exponential phase of pandemic. Journal of Clinical Investigation, 130(9), 4726-4733. https://doi.org/10.1172/JCI139587

Cheung, J. C. H., Ho, L. T., Cheng, J. V., Cham, E. Y. K., \& Lam, K. N. (2020). Staff safety during emergency airway management for COVID-19 in Hong Kong. The Lancet Respiratory Medicine, 8(4), e19. https://doi.org/10.1016/S2213-2600(20)30084-9

El-Boghdadly, K., Wong, D. J. N., Owen, R., Neuman, M. D., Pocock, S., Carlisle, J. B., Johnstone, C., Andruszkiewicz, P., Baker, P. A., Biccard, B. M., Bryson, G. L., Chan, M. T. V., Cheng, M. H., Chin, K. J., Coburn, M., Jonsson Fagerlund, M., Myatra, S. N., Myles, P. S., O’Sullivan, E., \& Ahmad, I. (2020). Risks to healthcare workers following tracheal intubation of patients with COVID-19: a prospective international multicentre cohort study. Anaesthesia, 75(11), 1437-1447. https://doi.org/10.1111/anae.15170

Feldman, O., Meir, M., Shavit, D., Idelman, R., \& Shavit, I. (2020). Exposure to a Surrogate Measure of Contamination From Simulated Patients by Emergency Department PersonnelWearing Personal Protective Equipment. JAMA, 323(20), 2091-2093. https://doi.org/10.1093/cid/ciaa330

Guo, L., Ren, L., Yang, S., Sharma, L., Wang, L., \& Wang, J. (2020). Profiling early humoral response to diagnose novel. Clinical Infectious Disease, 1-28.

Kim, H. J., Ko, J. S., \& Kim, T. Y. (2020). Recommendations for anesthesia in patients suspected of covid-19 coronavirus infection. Korean Journal of Anesthesiology, 73(2), 89-91. https://doi.org/10.4097/kja.20110

Lange, V. R. (2014). Eyewear contamination levels in the operating room: Infection risk. American Journal of Infection Control, 42(4), 446-447. https://doi.org/10.1016/j.ajic.2013.10.015

Lee, H., Jeong, S., Choi, C., Jeong, H., Lee, S., \& Jeong, S. (2013). Anesthesiologist's satisfaction using between cisatracurium and rocuronium for the intubation in the anesthesia induced by remifentanil and propofol. Korean Journal of Anesthesiology, 64(1), 34-39. https://doi.org/10.4097/kjae.2013.64.1.34

Lee, S. Y., Choi, S. H., Park, J. E., Hwang, S., \& Kwon, K. T. (2020). Crucial role of temporary airborne infection isolation rooms in an intensive care unit: Containing the COVID-19 outbreak in South Korea. Critical Care, 24(1), 4-6. https://doi.org/10.1186/s13054-020-02944-0

Lieberman, J. A., Pepper, G., Naccache, S. N., Huang, M. L., Jerome, K. R., \& Greninger, A. L. (2020). Comparison of commercially available and laboratorydeveloped assays for in vitro detection of sars-cov-2 in clinical laboratories. Journal of Clinical Microbiology, 58(8). https://doi.org/10.1128/JCM.00821-20

Loeffelholz, M. J., Alland, D., Butler-Wu, S. M., Pandey, U., Perno, C. F., Nava, A., Carroll, K. C., Mostafa, H., Davies, E., McEwan, A., Rakeman, J. L., Fowler, R. C., Pawlotsky, J. M., Fourati, S., Banik, S., Banada, P. P., Swaminathan, S., Chakravorty, S., Kwiatkowski, R. W., \& Persing, D. H. (2020). Multicenter evaluation of the cepheid xpert xpress sars-cov-2 test. Journal of Clinical Microbiology, 58(8). https://doi.org/10.1128/JCM.00926-20

London, M. J. (2020). Coronavirus disease 2019 (COVID-19): Anesthetic concerns, including airway management and infection control. UpToDate. https://www.uptodate.com/contents/coronavirus-disease-2019-covid-19-anesthetic-concerns-including-airway-management-and-infection-control/print

Magno, L., Rossi, T. A., de Mendonça-Lima, F. W., dos Santos, C. C., Campos, G. B., Marques, L. M., Pereira, M., Prado, N. M. de B. L., \& Dourado, I. (2020). Challenges and proposals for scaling up COVID-19 testing and diagnosis in Brazil. Ciencia e Saude Coletiva, 25(9), 3355-3364. https://doi.org/10.1590/141381232020259.17812020

Meng, L., Qiu, H., Wan, L., Ai, Y., Xue, Z., Guo, Q., Deshpande, R., Zhang, L., Meng, J., Tong, C., Liu, H., \& Xiong, L. (2020). Intubation and Ventilation amid the COVID-19 Outbreak: Wuhan's Experience. Anesthesiology, 6, 1317-1332. https://doi.org/10.1097/ALN.0000000000003296

Mercado Velázquez, P., Acosta Nava, V., Baltazar Torres, J. Á., Hernández Oropeza, J. L., Rivero Sigarroa, E., \& Domínguez Cherit, G. (2021). Manejo avanzado de la vía aérea en pacientes con sospecha o diagnóstico de COVID-19. Medicina Crítica, 35(1), 47-53. https://doi.org/10.35366/99153

Ministério da Saúde. (2020). Diretrizes para Diagnóstico e Tratamento da COVID-19. Secretaria De Ciência, Tecnologia, Inovação E Insumos Estratégicos Em Saúde, 1-398.

Offeddu, V., Yung, C. F., Low, M. S. F., \& Tam, C. C. (2017). Effectiveness of masks and respirators against respiratory infections in healthcare workers: a systematic review and meta-analysis. Clinical Infectious Diseasesct Dis, 65(11), 1934-1942.

Pereira A. S. et al. (2018). Metodologia da pesquisa científica. UFSM

Petherick, A. (2020). Developing antibody tests for SARS-CoV-2. Lancet (London, England), 395(10230), 1101-1102. https://doi.org/10.1016/S0140$6736(20) 30788-1$ 
Ruetzler, K., Smereka, J., Ludwin, K., Drozd, A., \& Szarpak, L. (2021). Respiratory protection among healthcare workers during cardiopulmonary resuscitation in COVID-19 patients. American Journal of Emergency Medicine, 39(April), 233. https://doi.org/10.1016/j.ajem.2020.05.014

Schumacher, J., Arlidge, J., Dudley, D., Sicinski, M., \& Ahmad, I. (2020). The impact of respiratory protective equipment on difficult airway management: a randomised, crossover, simulation study. Anaesthesia, 75(10), 1301-1306. https://doi.org/10.1111/anae.15102

Seresirikachorn, K., Phoophiboon, V., Chobarporn, T., Tiankanon, K., Aeumjaturapat, S., Chusakul, S., \& Snidvongs, K. (2021). Decontamination and reuse of surgical masks and N95 filtering facepiece respirators during the COVID-19 pandemic: A systematic review. Infection Control and Hospital Epidemiology, 42(1), 25-30. https://doi.org/10.1017/ice.2020.379

Tang, L. Y., \& Wang, J. (2020). Anesthesia and COVID-19: What We Should Know and What We Should Do. Seminars in Cardiothoracic and Vascular Anesthesia, 24(2), 127-137. https://doi.org/10.1177/1089253220921590

Vandenberg, O., Martiny, D., Rochas, O., van Belkum, A., \& Kozlakidis, Z. (2021). Considerations for diagnostic COVID-19 tests. Nature Reviews Microbiology, 19(3), 171-183. https://doi.org/10.1038/s41579-020-00461-z

Wang, D., Hu, B., Hu, C., Zhu, F., Liu, X., Zhang, J., Wang, B., Xiang, H., Cheng, Z., Xiong, Y., Zhao, Y., Li, Y., Wang, X., \& Peng, Z. (2020). Clinical Characteristics of 138 Hospitalized Patients with 2019 Novel Coronavirus-Infected Pneumonia in Wuhan, China. JAMA - Journal of the American Medical Association, 323(11), 1061-1069. https://doi.org/10.1001/jama.2020.1585

Wiersinga, W. J., Rhodes, A., Cheng, A. C., Peacock, S. J., \& Prescott, H. C. (2020). Pathophysiology, Transmission, Diagnosis, and Treatment of Coronavirus Disease 2019 (COVID-19): A Review. JAMA - Journal of the American Medical Association, 324(8), 782-793. https://doi.org/10.1001/jama.2020.12839

Zhu, N., Zhang, D., Wang, W., Li, X., Yang, B., Song, J., Zhao, X., Huang, B., Shi, W., Lu, R., Niu, P., Zhan, F., Ma, X., Wang, D., Xu, W., Wu, G., Gao, G. F., \& Tan, W. (2020). A Novel Coronavirus from Patients with Pneumonia in China, 2019. New England Journal of Medicine, 382(8), 727-733. https://doi.org/10.1056/nejmoa2001017. 